Oral presentation

\title{
OP-002 IMPROVEMENT OF GLYCEMIC CONTROL IN PREDIABETIC AND TYPE 2 DIABETIC PATIENTS UNDERGOING LAPAROSCOPIC SLEEVE GASTRECTOMY (LSG) IN NEWFOUNDLAND AND LABRADOR
}

Kendra Lester, Laurie Twells, Deborah Gregory, Christopher S. Kovacs, J.M. Gamble. Memorial University of Newfoundland

10.1136/jech-2013-203098.8

Introduction Newfoundland and Labrador (NL) has the highest prevalence of diabetes in the country. In 2011, 11.1\% of the population were identified as diabetic. LSG is a minimally invasive and irreversible procedure in which $80 \%$ of the stomach is removed, leaving a much smaller stomach or "sleeve". It is a promising treatment option for obesity, and recent studies demonstrate that LSG may be effective in improving/resolving T2DM in obese patients.

Objectives This study is evaluating LSG and its effectiveness in the improvement of glycemic control in prediabetes or type 2 diabetes mellitus (T2DM).

Methods All patients $(n=66)$ undergoing LSG in NL since May 2011 are followed prospectively. Patients are classified as having 
prediabetes (HbA1c: $5.7-6.4 \%$ or FPG: $6.1-6.9 \mathrm{mmol} / \mathrm{L}$ ) or T2DM (HbA1c $>6.5 \%$ or $\mathrm{FPG} \geq 7.0 \mathrm{mmol} / \mathrm{L}$, self-reported, or antidiabetic medication use) at baseline. Glycemic control is evaluated at 3, 6, and 12 months. The primary outcome is the proportion of patients experiencing improved glycemic control.

Results At baseline, $16(24.2 \%)$ patients have prediabetes (average HbA1c 5.8\% (SD 0.3), FPG $5.6 \mathrm{mmol} / \mathrm{L}$ (SD 0.7)) and 31 (47.0\%) have T2DM (average HbA1c 7.6\% (SD 1.4), FPG $8.1 \mathrm{mmol} / \mathrm{L}$ (SD 2.5)). Interim analysis within 6 months postsurgery: of the 11 prediabetic patients who've completed follow-up, $8(72.7 \%)$ have HbA1c $<5.7 \%(p=0.004)$ and 11 (100\%) $\mathrm{FPG}<6.1 \mathrm{mmol} / \mathrm{L}$; of T2DM patients who've completed follow-up, 12 of $19(63.2 \%)$ have $\mathrm{HbA} 1 \mathrm{c}<6.5 \%$ $(\mathrm{p}<0.001)$ and 14 of $17(82.4 \%)$ have $\mathrm{FPG}<7.0 \mathrm{mmol} / \mathrm{L}$ $(p=0.003)$. Prediabetes and T2DM groups show significant improvements in HbA1c $(-0.6 \%$ and $-1.5 \%$ respectively) and FPG $(-1.0 \mathrm{mmol} / \mathrm{L}$ and $-2.0 \mathrm{mmol} / \mathrm{L}$ respectively $), \mathrm{p}<0.001$ for all. 9 of 21 T2DM patients (42.9\%) who completed follow-up are no longer taking antidiabetic medications.

Conclusions Interim analysis indicates better glucose control is achieved within 6 months following LSG. 